\title{
Use of Event Detection Approaches for Outlier Detection in Wireless Sensor Networks
}

\author{
Majid Bahrepour, Yang Zhang, Nirvana Meratnia, Paul J.M. Havinga \\ Pervasive Systems Group, University of Twente, the Netherlands \\ (m.bahrepour, y.zhang, n.meratnia, p.j.m.havinga) @utwente.nl
}

\begin{abstract}
Outliers or anomalies are generally considered to be those observations that are considerably diverged from normal pattern of data. Due to their special characteristics, e.g. constrained available resources, frequent physical failure, and often harsh deployment area, wireless sensor networks (WSNs) are more likely to generate outliers compared to their other wireless counterparts. Potential sources of deviated data in a series of measurements are errors, events, and/or malicious attacks on the network. Current studies tend to handle events and errors separately and propose different techniques for event detection as for outlier detection. By bringing the concept of outlier and event close together and assuming that events are some sorts of outliers, in this paper, we investigate applicability of pattern matching-based event detection techniques for outlier detection. Through extensive experiments, we evaluate performance of various event detection techniques to detect outliers and compare them with a recent outlier detection study.
\end{abstract}

\section{INTRODUCTION}

Compared to wired networks, wireless sensor networks (WSNs), comprised of a large number of tiny, low-cost sensor nodes, equipped with sensing, computational power and shortrange wireless communication capabilities, have strong resource constraints in terms of energy, memory, computational capacity and communication bandwidth. The large-scale and high density vision of the WSN suggests that the network usually has to operate in a harsh and unattended environment. Moreover WSNs are vulnerable to faults and malicious attacks (e.g., denial of service attacks or black hole attacks), and cause unreliable and inaccurate sensor readings. Generally speaking, the potential sources of outliers include noise and errors, events, and malicious attacks on the network. To ensure a reasonable data quality, secure monitoring and reliable detection of interesting and critical events, and to facilitate effective and correct decision-making using data collected by WSNs, identifying anomalous measurements in point of action is a must.

In WSNs, outliers, are those measurements that do not conform to the normal behavioral pattern of the sensed data [1]. Traditional outlier detection techniques are not directly applicable to wireless sensor networks due to specific requirements, dynamic nature, and limitations of the wireless sensor networks [28]. An appropriate outlier detection technique for the WSN should pay attention to computing, communication and storage limitations of the network and deal with the distributed data analysis. The key objective of outlier detection in WSNs is to identify outliers in the distributed streaming data in an online manner with a high accuracy while maintaining the resource consumption of the network to a minimum.

From data mining point of view, outlier detection is a machine learning job, in which outliers should be distinguished from normal data. It is this perspective that makes outlier detection in line with event detection or any other pattern matching task.

Distinguishing between causes of outliers is important as it gives an insight on how to handle the detected outlier. Simply speaking if the detected outlier is not an event, it should be removed from the sensory data to ensure high data quality and save energy by eliminating communication load. On the other hand, if the outlier is caused by an event (e.g. fire), removing the outlier will lead to information loss, which may have undesired consequences. Errors in WSNs may occur because human errors, mechanical faults, or change in the environment. Current research tends to deal with outliers and events separately and proposes different techniques for those without investigating the applicability of one for another. Therefore, by considering the fact that events are some sorts of outliers (as events are one of the causes of outliers), in this paper we investigate how well event detection techniques can identify outliers.

The remainder of this paper is organized as follows. Section II provides an overview of previous studies in both fields of outlier and event detection. Section III introduces the event detection techniques, which are going to be used for outlier detection in this study. Section IV presents experiments, data analysis, comparisons of the event detection and outlier detection techniques, and lessons learned. Conclusion and directions for future study will be addressed in Section V.

\section{LITERATURE SURVEY}

Generally speaking, outlier detection techniques for WSNs can be categorized into statistical-based, nearest neighborbased, clustering-based, classification-based and spectral decomposition-based approaches [1, 4]. Statistical-based approaches are further categorized into parametric [5] and non-parametric [6] approaches based on how the probability distribution model is built. They assume or estimate a statistical (probability distribution) model which captures the distribution of the data and evaluate data instances with respect to how well they fit the model. A data instance is declared as an outlier if the probability of the data instance to be generated by this model is very low. Nearest neighborbased approaches [5,7] use several well-defined distance 
notions to compute the distance (similarity measure) between two data instance. A data instance is declared as an outlier if it is located far from its neighbors. Clustering-based approaches $[8,9]$ are popular approaches within the data mining community to group similar data instances into clusters with similar behavior. Data instances are identified as outliers if they do not belong to clusters or if their clusters are significantly smaller than other clusters. Classification-based approaches are categorized as Bayesian network-based [10, $11]$, neural network-based $[2,12]$ and support vector machinebased [13, 14] approaches based on type of classification model they use. They learn a classification model using a set of data instances in the training phase and classify an unseen instance into one of the learned (normal/outlier) class in the testing phase. Spectral decomposition-based approaches aim at finding normal modes of behavior in the data by using principle components [15].

Event detection, on the other hand, has been tackled from a different perspective. In some studies events are detected using a user-defined threshold [16-18]. In such approaches an event alarm is arisen when sensor readings are lower or higher than a predefined threshold value. To cope with shortcomings of pre-defined thresholds, some techniques such as the one proposed by Liang and Wang present a dynamic threshold approach in which the threshold value is found dynamically using a slide window method [19]. The new trend in event detection is using pattern matching techniques, in which the pattern matching itself may be performed in a base station [20, 21], locally on the sensor nodes [2], or collaboratively between some sensor nodes in a region [3, 12, 22-24]. Martincic and Schwiebert [24] employ a cell-based network architecture to locally detect events based on collaboration among neighboring nodes. Luo et al. [23] take into account different level of sensor fault probability during event detection. Krishnamachari and Iyengar [12] propose a distributed Bayesian protocol to detect event regions in presence of faulty sensors. Ding et al. [25] attempt to identify event boundaries since detection of event boundary may become more important than detection of event region because of unreliability of sensor measurements. A distributed fuzzy engine has also been used for event detection [26].

\section{USE OF EVENT DETECTION FOR OUTLIER DETECTION}

In this section, we aim at bridging the gap between two traditionally separated domains of event and outlier detection. The basic idea we explore here is that although errors and events are semantically different and different mechanisms cause them, they both are conceptually the same, i.e., measurements than are significantly different from the rest of the observations or normal pattern of data. A distinguishing factor between the two, however, is that errors are more local and node dependent, while events have more global influence and have effects on neighboring nodes as well. By considering events as outliers, we investigate how well event detection approaches can distinguish outliers. To do so, we analyze four machine-learning techniques, i.e., Naïve Bayes, Feed Forward Neural Network, standard support vector machine, and our proposed fusion-based approach [2, 3] and compare their performance with an optimal outlier detection technique recently proposed in [4], i.e., Quarter-Sphere SVM.

We continue this section by presenting basic principles and challenges introduced by the techniques used.

\section{A. Feed Forward Neural Network \& Naïve Bayes}

An artificial neural network (ANN) is a computational model based on organic neural networks. It consists of an interconnected collection of artificial neurons processes information using a connectionist method [2, 29]. As a simple modeling of biological neural networks, feed forward neural network (FFNN) consists of one input layer, one or more hidden layers and one output layer. Inputs are the data and the output is a predicted value (class) that input data is expected to belong to.

The Naïve Bayes classifier uses Bayesian statistics and Bayes theorem to find the probability of each instance belonging to a specific class. It is called Naïve because of its emphasis on independency of the input data [2]. To find the probability of belongingness of each instant to a specific class, Eq. 1 is used, that expresses the probability of an example $E=\left(x_{1}, x_{2}, \ldots, x_{n}\right)$ belonging to class $c$ [2].

$p(c \mid E)=\frac{p(E \mid c) p(c)}{p(E)}$

Naïve Bayes and FFNN are considered suitable for event detection (or generally, pattern matching) in WSNs due to the fact that they are accurate and computationally light and, therefore, can easily be implemented on sensor nodes [2, 3] and be simply evaluated as business rules. The challenge faced here, however, is the computationally expensive training part. We previously showed that training them offline, learning the model, and translating it to simple mathematical formulas running on the sensor nodes enable the wireless sensor network community to benefit from the powerful pattern matching and classification capability of these techniques $[2,3]$. We use the same approach in this paper as well.

\section{B. Standard Support Vector Machine (SVM)}

Support vector machine (SVM)-based techniques are from a family of classification-based approaches. The main idea of these techniques is to separate the data belonging to different classes by fitting a hyperplane that produces a maximal margin. They have three main advantages for classification: (a) do not require an explicit statistical model; (b) provide an optimum solution for classification by maximizing the margin of the decision boundary; (c) avoid the curse of the dimensionality problem.

One of the challenges of SVM-based outlier detection techniques for WSNs is obtaining error-free or labeled data for training. One-class (unsupervised) SVM-based techniques address this challenge by modeling normal behavior of the unlabelled data while automatically ignoring the anomalies existing in the training set. They use a non-linear function to map the data vectors collected from the original space to a higher dimensional space called feature space. Then a 
decision boundary is found, which encompasses the majority of the data vectors in the feature space. Those new data vectors falling outside this decision boundary are classified as outliers.

In addition to obtaining the labeled data, another challenge faced by SVM-based techniques is the inapplicability of their quadratic optimization during the learning process for WSN applications. This process is extremely costly and unsuitable for limited resources available in WSNs. To deal with this problem, Laskov et al. [27] propose a one-class quarter-sphere SVM, which is formulated as a linear optimization problem and thus reduces the effort and computational complexity.

\section{Quarter-Sphere SVM}

We previously extended the one-class quarter-sphere SVMbased technique of Laskov et al. [27] and proposed a distributed and online outlier detection to identify every new measurement collected at each node as normal or anomalous in real-time [28]. The geometry of the one-class quartersphere SVM is shown in Fig. 1.

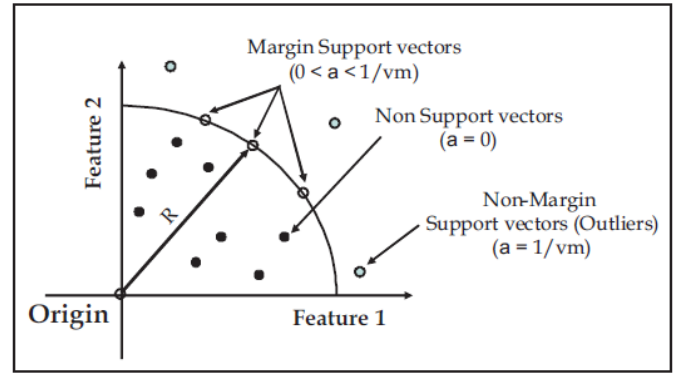

Fig. 1: Geometry of the quarter-sphere formulation of one-class SVM [27]

Our proposed technique is based on the assumptions that sensor nodes are time synchronized and are densely deployed in a homogeneous WSN, where sensor data tends to be correlated in both time and space. In this situation, each sensor node in the network is able to exploit temporal correlations among its most recent sensor measurements to identify its new arriving measurement as normal or anomalous in real-time. Moreover, using high-degree spatial correlations that exist between sensor readings of adjacent nodes, each node has more information to cooperatively identify outliers. The whole detection process not only depends on a node's own decision criterion learned from its temporal readings but also on the decision criteria learned from its neighboring nodes.

Initially, each node learns the local radius of the quartersphere using its $m$ sequential data measurements, which may include some anomalous data. The one-class quarter-sphere SVM can efficiently find a minimal radius $R$ to enclose the majority of these mapped sensor measurements in the feature space. Each node then locally broadcasts the learned radius information to its neighboring nodes. Upon receiving the radii from all of its neighbors, each node computes a median radius $R_{m}$ of its neighboring nodes in the sub-network. When a new sensor measurement $x_{t}$ is collected at time $t$, the node first compares the distance of $x_{t}$ from the origin with the radius $R$ learned with respect to its $m$ previous measurements The measurement $x_{t}$ will be classified as normal if $d\left(x_{t}\right)<=R$, which means that $x_{t}$ falls on or inside the quarter-sphere. Otherwise if $d\left(x_{t}\right)>R$, the node further compares $d\left(x_{t}\right)$ with the median radius $R_{m}$ of its spatially neighboring nodes. Then if $d\left(x_{t}\right)>R_{m}, x_{t}$ will finally be classified as outlier in the subnetwork.

\section{Fusion based Feed Forward Neural Network \& Nä̈ve Bayes}

The fusion-based approach, which we originally proposed for distributed event detection, uses the FFNN and Bayes classifiers in two phases [3]. As illustrated in Fig. 2, the first phase of classification is performed locally on each sensor node, while in the second phase, performed in a cluster head, or an aggregator node, outcomes of the first classification are aggregated. To support heterogeneous networks, nodes are not considered to have exact same sensor types. Flexibility of the approach also allows use of different classifiers in the two levels and selection of the best combination, which achieves highest classification accuracy and lowest false positives.

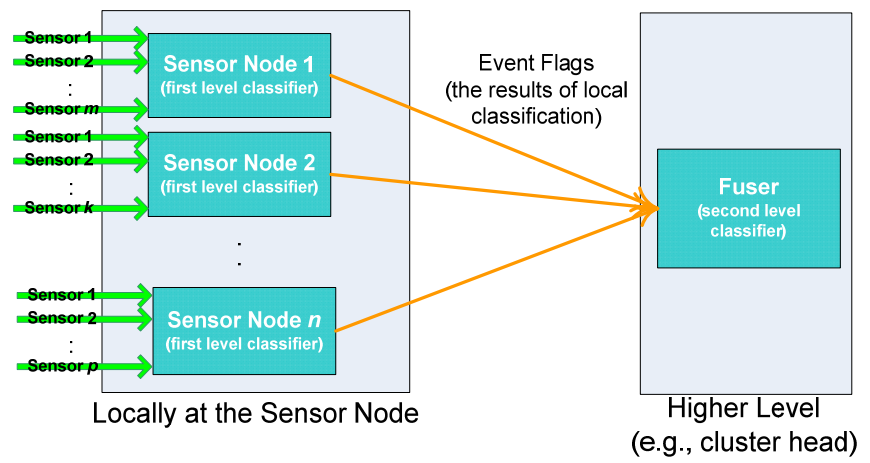

Fig. 2: General Concept of our Fusion-based Approach

\section{EXPERIMENTAL STUDIES}

To evaluate suitability of event detection techniques for outlier detection, we perform several experiments in Matlab ${ }^{\circledR}$, in which temperature and relative humidity data are given to different classifiers mentioned in previous section and their performance in terms of both outlier detection rate and false positive rate is analyzed.

For Naïve Bayes classifier, the data distribution is calculated using a histogram approach. Therefore, we assume neither a normal nor well-known data distribution. To obtain the histogram, we partition the whole dataset into $m$ intervals. The appropriate $m$ value is found through extensive experiments.

FFNN is also trained offline and the optimal topologies as well as the weights are acquired in an offline process.

\section{E. Synthetic and Real Datasets}

In our experiments, we use both synthetic and real datasets. The size of dataset is 220 instances per each sensor node for synthetic data and 1200 for the real data. For the simulation, we consider a sensor sub-network consisting of 7 sensor nodes, which are within radio transmission range of each other. This 
implies that each node in this sub-network has 6 neighboring nodes.

The 2-D synthetic data used for each node is composed of a mixture of three Gaussian distribution with uniform outliers; the mean is randomly selected from $(0.3,0.35,0.45)$, and the standard deviation is selected as 0.03 . Subsequently, $10 \%$ (of the normal data) anomalous data is introduced and uniformly distributed in the interval $[0.5,1]$. The data values are normalized to fit in the $[0,1]$. The testing data used for each node comprises of 200 normal and 20 anomalous data. Fig. 3 illustrates data distribution of the synthetic data.

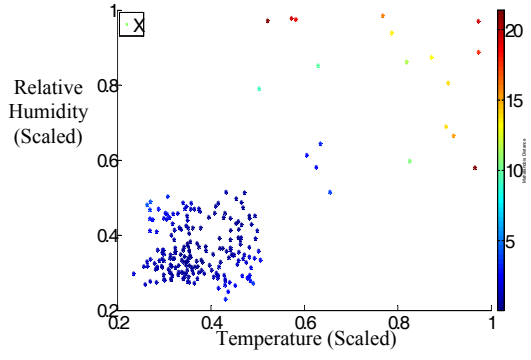

Fig. 3: Scattered plot of synthetic data

The real data, on the other hand, is collected from a closed neighborhood from a WSN deployed in Grand-St-Bernard, Switzerland [30]. The network records ambient temperature, relative humidity, soil moisture, solar radiation and watermark measurements at every two minutes interval. In our experiments, we use a cluster of data recorded on four following days with two attributes: ambient temperature and relative humidity for each sensor measurement. Each data point is labeled as either normal or outlier based on dissimilarity degree. We use Mahalanobis distance to identify data measurements distant from the other data vectors and labeled them as outliers.

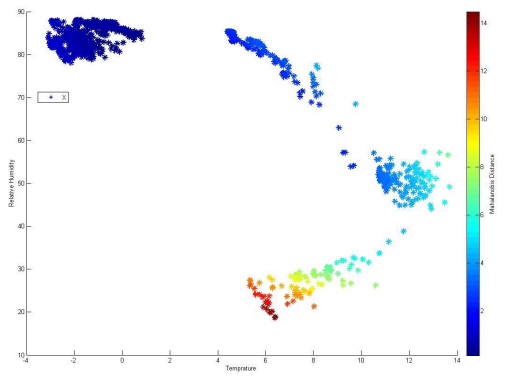

Fig. 4: Scattered plot of real data collected from the Grand-St-Bernard deployment by SensorScope System [30]

\section{F. Experimental Results on Synthetic Data}

To perform the experiment, seven datasets from seven sensor nodes are chosen. Both Naïve Bayes and FFNN classifiers are trained by $(2 / 3)$ of the data and tested by the other (1/3). Each sensor node had 220 records, each of which containing temperature and relative humidity data. For each dataset related to the sensor nodes the experiments are repeated 10 times and the average values are calculated.
The best parameters and the best accuracy results for Naïve Bayes and FFNN are given in Tables I.

TABLE I

THE ACCURACY RESULTS FOR NAÏVE BAYES AND FFNN

\begin{tabular}{|l|l|l|l|}
\hline Method & $\begin{array}{l}\text { Detection } \\
\text { Rate }\end{array}$ & \multicolumn{1}{|c|}{$\begin{array}{l}\text { False } \\
\text { Positive }\end{array}$} \\
\hline Naïve Bayes & $94.84 \%$ & $0 \%$ & 10 intervals \\
\hline FFNN & $96.95 \%$ & $0.32 \%$ & $\begin{array}{l}40 \text { neurons in the } \\
\text { hidden layer }\end{array}$ \\
\hline $\begin{array}{l}\text { The Fusion- } \\
\text { based approach } \\
\text { (using Naïve } \\
\text { Bayes) }\end{array}$ & $84.9 \%$ & $2.1 \%$ & $\begin{array}{l}8 \text { intervals for the first } \\
\text { level, 2000 for the } \\
\text { second level }\end{array}$ \\
\hline $\begin{array}{l}\text { The Fusion- } \\
\text { based approach } \\
\text { (using FFNN) }\end{array}$ & $85.95 \%$ & $0.06 \%$ & $\begin{array}{l}\text { 150 neuron in the } \\
\text { hidden layer for the } \\
\text { fist level and 300 } \\
\text { neuron for the second } \\
\text { level }\end{array}$ \\
\hline
\end{tabular}

Detection rate represents number of detected outliers that have actually been outliers, while false positives represents number of normal data points detected as outlier.

To analyze the fusion-based approaches, training and testing should be carried out somewhat differently. The first level of classification is trained first and then the generated outputs are used for training the second level. Therefore, we split the whole data into (2/3) and (1/3) for training and testing, respectively. Then, we split the $(1 / 3)$ of the test results obtained in the first level of classification again into another $(2 / 3)$ and (1/3) for training and testing for the second level.

The parameters and the best results achieved using fusionbased approaches are reported in Table I.

Training and testing of the standard SVM are performed similarly to the Naïve Bayes and FFNN. Training and testing of Quarter-Sphere SVM, on the other hand, is done according to [4]. The parameters and the best results achieved by these two techniques are reported in Table II.

TABLE II

THE ACCURACY RESULTS OF THE STANDARD SVM AND QUARTER-SPHERE SVM

\begin{tabular}{|l|l|l|l|}
\hline Method & $\begin{array}{l}\text { Detection } \\
\text { Rate }\end{array}$ & \multicolumn{1}{c|}{$\begin{array}{l}\text { False } \\
\text { Positive }\end{array}$} & Parameters \\
\hline Standard SVM & $98.12 \%$ & $0.19 \%$ & $\begin{array}{l}\text { Using polynomial } \\
\text { kernel function }\end{array}$ \\
\hline $\begin{array}{l}\text { Quarter-Sphere } \\
\text { SVM [4] }\end{array}$ & $98.53 \%$ & $1.58 \%$ & $\begin{array}{l}\text { Using polynomial } \\
\text { kernel function }\end{array}$ \\
\hline
\end{tabular}

\section{G. Experimental Results on Real Data}

The experiments discussed in the previous subsection were conducted on synthetic data. Table III presents, however, a side study on real data. The real dataset contains 1200 data records, out of which around $8 \%$ are outliers and $92 \%$ normal data. Subsection E describes the data. Each experiment is repeated 10 times and the average values are reported. 
TABLE III

THE RESULTS ON REAL DATA DATASET

\begin{tabular}{|c|c|c|c|}
\hline Method & $\begin{array}{l}\text { Detection } \\
\text { Rate }\end{array}$ & $\begin{array}{l}\text { False } \\
\text { Positive }\end{array}$ & Parameters \\
\hline Naïve Bayes & $75.07 \%$ & $0.78 \%$ & 80 intervals \\
\hline FFNN & $96.04 \%$ & $0.38 \%$ & $\begin{array}{l}40 \text { neurons in the } \\
\text { hidden layer }\end{array}$ \\
\hline $\begin{array}{l}\text { The Fusion- } \\
\text { based approach } \\
\text { (using Naïve } \\
\text { Bayes) }\end{array}$ & $91 \%$ & $12.13 \%$ & $\begin{array}{l}80 \text { intervals in the } \\
\text { first level and } 2000 \\
\text { in the second level }\end{array}$ \\
\hline $\begin{array}{l}\text { The Fusion- } \\
\text { based approach } \\
\text { (using FFNN) }\end{array}$ & $92.01 \%$ & $0.085 \%$ & $\begin{array}{l}40 \text { neurons in } \\
\text { hidden layer of the } \\
\text { first level and } 60 \text { in } \\
\text { the second level }\end{array}$ \\
\hline
\end{tabular}

The parameters and the best results achieved by both standard and Quarter-Sphere SVM are reported in Table IV.

TABLE IV

THE ACCURACY RESULTS OF

THE STANDARD SVM AND QUARTER-SPHERE SVM

\begin{tabular}{|l|l|l|l|}
\hline Method & $\begin{array}{l}\text { Detection } \\
\text { Rate }\end{array}$ & $\begin{array}{l}\text { False } \\
\text { Positive }\end{array}$ & Parameters \\
\hline Standard SVM & $97.64 \%$ & $0.21 \%$ & $\begin{array}{l}\text { Using polynomial } \\
\text { kernel function }\end{array}$ \\
\hline $\begin{array}{l}\text { Quarter-Sphere } \\
\text { SVM [4] }\end{array}$ & $98.05 \%$ & $1.24 \%$ & $\begin{array}{l}\text { Using polynomial } \\
\text { kernel function }\end{array}$ \\
\hline
\end{tabular}

\section{H. Computational Complexity}

Besides the detection rate, computational complexity and memory size implication are also key factors for comparison. Table V summarizes time complexities of the presented approaches. Memory complexity is out of scope of this paper.

From Table V, it can be concluded that, Quarter-Sphere SVM, FFNN, Naïve Bayes and the fusion-based FFNN are lighter algorithms.

\section{Lessons Learned}

Table VI summarizes our experimental results on the performance evaluation of event detection techniques for detecting outliers.

An important lesson learned is that a few data records in the training phase probably leads to poor classification results in the testing phase in the fusion-based approaches. This effect can clearly be seen from the experimental results on the synthetic data. The 220 instances available in the synthetic dataset are clearly insufficient for training the complicated fusion-based approaches. Being trained with enough data (for example 1200 instances of the real data), however, results in far better outcome. For future works we will elaborate on this.

Another lesson learned is that to identify outliers locally on the nodes, among the available techniques, i.e., FFNN, Naïve Bayes, and standard SVM, the latter achieves the best detection rate. However, it is the most expensive technique in terms of computational cost. Therefore, FFNN seems to be the right technique to be used. From reported results it can be seen this event detection technique has similar level of performance as the Quarter-Sphere SVM outlier detection.
Finally, while use of fusion-based approaches lead to better detection accuracy compared to the local approaches, their higher communication overhead is may be a concern.

TABLE V

COMPUTATIONAL COMPLEXITY OF THE APPROACHES

\begin{tabular}{|c|c|c|}
\hline Method & $\begin{array}{l}\text { Computational } \\
\text { Complexity }\end{array}$ & Comment \\
\hline Naïve Bayes & $O_{N B}=O(m \times i \times j)$ & $\begin{array}{l}\text { Where } \boldsymbol{m} \text { is number of } \\
\text { features, } \boldsymbol{i} \text { is number of } \\
\text { classes, and } \boldsymbol{j} \text { is number } \\
\text { of intervals }\end{array}$ \\
\hline FFNN & $O_{F F N N}=O(m \times n)$ & $\begin{array}{l}\text { Where } \boldsymbol{m} \text { is number of } \\
\text { neurons in input layer } \\
\text { (number of features), } \boldsymbol{n} \\
\text { is number of neurons in } \\
\text { hidden layer. }\end{array}$ \\
\hline $\begin{array}{l}\text { The Fusion- } \\
\text { based approach } \\
\text { (using Naïve } \\
\text { Bayes) }\end{array}$ & $O_{T N B}=O(k \times m \times i \times j)$ & $\begin{array}{l}\text { Where } \boldsymbol{k} \text { is the number } \\
\text { of nodes that run the } \\
\text { algorithm in parallel (in } \\
\text { the case of outlier } \\
\text { detection } \boldsymbol{k}=3 \text { ). }\end{array}$ \\
\hline $\begin{array}{l}\text { The Fusion- } \\
\text { based approach } \\
\text { (using FFNN) }\end{array}$ & $O_{T F F N N}=O(k \times m \times n)$ & $\begin{array}{l}\text { Where } \boldsymbol{k} \text { is the number } \\
\text { of nodes that run the } \\
\text { algorithm in parallel (in } \\
\text { the case of outlier } \\
\text { detection } \boldsymbol{k}=3 \text { ). }\end{array}$ \\
\hline Standard SVM & $O_{S V M}=O(m \times p)$ & $\begin{array}{l}\text { Where } \boldsymbol{m} \text { is number of } \\
\text { features, } \boldsymbol{p} \text { is number of } \\
\text { data vectors (number of } \\
\text { classes). }\end{array}$ \\
\hline $\begin{array}{l}\text { Quarter-Sphere } \\
\text { SVM }\end{array}$ & $O_{Q S V M}=O(m \times p)$ & $\begin{array}{l}\text { Where } \boldsymbol{m} \text { is number of } \\
\text { features, } \boldsymbol{p} \text { is number of } \\
\text { data vectors (number of } \\
\text { classes). }\end{array}$ \\
\hline
\end{tabular}

TABLE VI

COMPARING DIFFERENT APPROACHES ON OUTLIER DATA

\begin{tabular}{ll|l|} 
Technique Name & $\begin{array}{l}\text { Accuracy Rate } \\
\text { On Synthetic } \\
\text { Data }\end{array}$ & $\begin{array}{l}\text { Accuracy Rate } \\
\text { On Real Data }\end{array}$ \\
Quarter-Sphere SVM & $98.53 \%$ & $98.05 \%$ \\
\hline Standard SVM & $98.12 \%$ & $97.64 \%$ \\
\hline FFNN & $96.95 \%$ & $96.04 \%$ \\
\hline $\begin{array}{l}\text { The Fusion-based Approach } \\
\text { (Naïve Bayes) }\end{array}$ & $84.9 \%$ & $91 \%$ \\
$\begin{array}{l}\text { The Fusion-based Approach } \\
\text { (FFNN) }\end{array}$ & $85.95 \%$ & $98.21 \%$ \\
\hline Naïve Bayes & $94.84 \%$ & $75.07 \%$ \\
\hline
\end{tabular}

\section{CONCLUSION}

In contrary to the current trend on event and outlier detection, which handles them separately, in this paper, we brought these two concepts close together. By considering events as some sorts of outliers, we have investigated applicability and suitability of pattern-matching event detection techniques for outlier detection. Our extensive experiments show that these techniques can be as good as outlier detection techniques. This implies that same techniques may be used for detecting both events and outliers. 
Among the techniques investigated in this paper, i.e., FFNN, Naïve Bayes, standard SVM, Quarter-Sphere SVM, and fusion-based techniques, Quarter-Sphere SVM and FFNN outperform the rest in terms of both detection accuracy and computational cost.

\section{ACKNOWLEDGMENT}

This work is supported by the EU Seventh Framework Programme, the SENSEI project.

\section{REFERENCES}

[1] V. Chandola, A. Banerjee, and V. Kumar, "Anomaly Detection - A Survey," ACM Computing Surveys, vol. 41, 2009.

[2] M. Bahrepour, N. Meratnia, and P. J. M. Havinga, "Use of AI Techniques for Residential Fire Detection in Wireless Sensor Networks," in AIAI 2009 Thessaloniki, Greece: ceur-ws.org, 2009.

[3] M. Bahrepour, N. Meratnia, and P. J. M. Havinga, "Sensor Fusionbased Event Detection in Wireless Sensor Networks," in MobiQuitous, Sensor Fusion 09 Workshop, 2009.

[4] Y. Zhang, N. Meratnia, and P. Havinga, "An online outlier detection technique for wireless sensor networks using unsupervised quartersphere support vector machine," in Fourth International Conference on Intelligent Sensors, Sensor Networks and Information Processing, ISSNIP 2008, Sydney, Australia, 2008, pp. 151-156.

[5] W. Wu, X. Cheng, M. Ding, K. Xing, and F. Liu, "Localized outlying and boundary data detection in sensor networks," IEEE Transactions on Knowledge and Data Engineering, vol. 19, pp. 1145-1157, 2007.

[6] S. Subramaniam, T. Palpanas, D. Papadopoulos, V. Kalogeraki, and D. Gunopulos, "Online outlier detection in sensor data using nonparametric models," in Proceedings of the 32nd international conference on Very large data bases, 2006, pp. 187-198.

[7] Y. Zhuang and L. Chen, "In-Network outlier cleaning for data collection in sensor networks," in Proceedings of CleanDB, Workshop in $V L D B, 2006$.

[8] S. Rajasegarar, C. Leckie, M. Palaniswami, and J. C. Bezdek, "Distributed anomaly detection in wireless sensor networks," in Proceedings of Communication systems, 2006, pp. 1-5.

[9] A. Lazarevic, A. Ozgur, L. Ertoz, J. Srivastava, and V. Kumar, "A comparative study of anomaly detection schemes in network intrusion detection," in Proceedings of SIAM, 2003.

[10] E. Elnahrawy and B. Nath, "Context-Aware sensors," in Proceedings of European Wireless Sensor Networks, 2004, pp. 77-93.

[11] D. Janakiram, A. Mallikarjuna, V. Reddy, and P. Kumar, "Outlier Detection in Wireless Sensor Networks using Bayesian Belief Networks," in IEEE Comsware, 2006.

[12] B. Krishnamachari and S. Iyengar, "Distributed Bayesian algorithms for fault-tolerant event region detection in wireless sensor networks," IEEE Transactions on Computers, vol. 53, pp. 241-250, 2004.

[13] S. Rajasegarar, C. Leckie, M. Palaniswami, and J. C. Bezdek, "Quarter Sphere based Distributed Anomaly Detection in Wireless Sensor Networks," in IEEE International Conference on Communications, Glasgow, England, 2007, pp. 3864-3869.

[14] Y. Zhang, N. Meratnia, and P. J. M. Havinga, "Adaptive and Online One-Class Support Vector Machine-based Outlier Detection Techniques for Wireless Sensor Networks.," in IEEE 23rd International Conference on Advanced Information Networking and Applications, 2009.

[15] V. Chatzigiannakis, S. Papavassiliou, M. Grammatikou, and B. Maglariset, "Hierarchical anomaly detection in distributed large-scale sensor networks," in Proceedings of Computers and Communications, 2006, pp. 761-767.

[16] M. L. Segal, F. P. Antonio, S. Elam, J. Erlenbach, and K. R. de Paolo, "Method and apparatus for automatic event detection in a wireless communication system," U. Patent, Ed. USA, 2000.

[17] C. T. Vu, R. A. Beyah, and Y. Li, "Composite Event Detection in Wireless Sensor Networks," in Performance, Computing, and Communications Conference, 2007. IPCCC 2007. IEEE Internationa, 2007.
[18] G. Werner-Allen, K. Lorincz, M. Ruiz, O. Marcillo, J. Johnson, J. Lees, and M. Welsh, "Deploying a wireless sensor network on an active volcano," IEEE Internet Computing, vol. 10, pp. 18-25, 2006.

[19] Qilian Liang and L. Wang, "Event detection in sensor networks using fuzzy logic system," in EEE Intl Conference on Computational Intelligence for Homeland Security and Personal Safety, Orlando, FL, 2005.

[20] D. Li, K. D. Wong, Y. H. Hu, and A. M. Sayeed, "Detection, classification, and tracking of targets," Signal Processing Magazine, IEEE, vol. 19, pp. 17-29, 2002.

[21] W. Xue, Q. Luo, L. Chen, and Y. Liu, "Contour map matching for event detection in sensor networks," in International Conference on Management of Data, Chicago, IL, USA 2006.

[22] G. Jin and S. Nittel, "NED: An Efficient Noise-Tolerant Event and Event Boundary Detection Algorithm in Wireless Sensor Networks," in 7th International Conference on Mobile Data Management, 2006.

[23] X. Luo, M. Dong, and Y. Huang, "On distributed fault-tolerant detection in wireless sensor networks," IEEE Transactions on Computers, vol. 55, pp. 58-70, 2006.

[24] F. Martincic and L. Schwiebert, "Distributed event detection in sensor networks," in Proceedings of the International Conference on Systems and Networks Communication, 2006, pp. 43-48.

[25] M. Ding, D. Chen, K. Xing, and X. Cheng, "Localized fault-tolerant event boundary detection in sensor networks," in Proceedings of IEEE Conference of Computer and Communications Societies, 2005, pp. 902-913.

[26] M. Marin-Perianu and P. J. M. Havinga, " D-FLER - A Distributed Fuzzy Logic Engine for Rule-Based Wireless Sensor Networks," Springer Verlag, Germany, 2007, pp. 86-101.

[27] P. Laskov, C. Schafer, and I. Kotenko, "Intrusion Detection in Unlabeled Data with Quarter Sphere Support Vector Machines," in Detection of Intrusions and Malware \& Vulnerability Assessment, Dortmund, Germany, 2004, pp. 71-82.

[28] Y. Zhang, N. Meratnia, and P. J. M. Havinga, "Outlier Detection Techniques for Wireless Sensor Networks: A Survey," International Journal of IEEE Communications Surveys and Tutorials, vol. Accepted for publication, 2009.

[29] Wikipedia, "Artificial neural network," March 2009.

[30] EPFL University, "EPFL SensorScope," http://sensorscope.epfl.ch/index.php/Main_Page, 2007. 